\title{
Vector network analyzer measurement of the amplitude of an electrically excited surface acoustic wave and validation by X-ray diffraction
}

I. S. Camara, B. Croset, L. Largeau, P. Rovillain, L. Thevenard, and J.-Y. Duquesne

Citation: Journal of Applied Physics 121, 044503 (2017); doi: 10.1063/1.4974947

View online: http://dx.doi.org/10.1063/1.4974947

View Table of Contents: http://aip.scitation.org/toc/jap/121/4

Published by the American Institute of Physics

\section{Articles you may be interested in}

An analysis of electric double layers near comb electrodes using the linearized Poisson-Nernst-Planck theory Journal of Applied Physics 121, 044502044502 (2017); 10.1063/1.4974468

Thermal transport at the nanoscale: A Fourier's law vs. phonon Boltzmann equation study

Journal of Applied Physics 121, 044302044302 (2017); 10.1063/1.4974872

Aggregation state and magnetic properties of magnetite nanoparticles controlled by an optimized silica coating Journal of Applied Physics 121, 044304044304 (2017); 10.1063/1.4974532

Fabrication of double barrier structures in single layer c-Si-QDs/a-SiOx films for realization of energy selective contacts for hot carrier solar cells

Journal of Applied Physics 121, 044305044305 (2017); 10.1063/1.4974739 


\title{
Vector network analyzer measurement of the amplitude of an electrically excited surface acoustic wave and validation by X-ray diffraction
}

\author{
I. S. Camara, ${ }^{1, a)}$ B. Croset,${ }^{1}$ L. Largeau, ${ }^{2}$ P. Rovillain, ${ }^{1}$ L. Thevenard, ${ }^{1}$ and J.-Y. Duquesne ${ }^{1}$ \\ ${ }^{1}$ Sorbonne Universités, UPMC Univ Paris 06, CNRS-UMR 7588, Institut des Nanosciences de Paris, \\ 75005 Paris, France \\ ${ }^{2}$ Centre de Nanosciences et de Nanotechnologies, CNRS, Univ. Paris-Sud, Université Paris-Saclay, \\ 91460 Marcoussis, France
}

(Received 13 October 2016; accepted 13 January 2017; published online 26 January 2017)

\begin{abstract}
Surface acoustic waves are used in magnetism to initiate magnetization switching, in microfluidics to control fluids and particles in lab-on-a-chip devices, and in quantum systems like two-dimensional electron gases, quantum dots, photonic cavities, and single carrier transport systems. For all these applications, an easy tool is highly needed to measure precisely the acoustic wave amplitude in order to understand the underlying physics and/or to optimize the device used to generate the acoustic waves. We present here a method to determine experimentally the amplitude of surface acoustic waves propagating on Gallium Arsenide generated by an interdigitated transducer. It relies on Vector Network Analyzer measurements of S parameters and modeling using the Coupling-Of-Modes theory. The displacements obtained are in excellent agreement with those measured by a very different method based on X-ray diffraction measurements. Published by AIP Publishing.
\end{abstract}

[http://dx.doi.org/10.1063/1.4974947]

\section{INTRODUCTION}

In recent years, the range of applications of surface acoustic waves (SAWs) has greatly increased. Excited electrically using interdigitated transducers (IDTs), they are routinely used as band pass filters in RF microelectronics up to $3 \mathrm{GHz},{ }^{1}$ in touch-sensitive screens and biological/chemical sensing. ${ }^{2-5}$ In more academic environments, they are used in magnetism, in microfluidics and in quantum systems. In magnetism, SAWs can assist magnetization switching in ferromagnetic magnetostrictive thin films and nanostructures $^{6-10}$ for magnetic data storage application. The efficiency of that switching is related to the amplitude of the SAW. Surface acoustic waves have also been proposed as an alternative method for synchronously propagating and pinning domain walls in magnetic nanowires for applications in racetrack memory devices. ${ }^{11,12}$ It was found that the velocity of propagation of the domain walls depends on the amplitude of the SAW. In the area of microfluidics, recent research demonstrates that SAWs provide an effective means to manipulate fluids (fluid mixing, ${ }^{13-15}$ translation, ${ }^{16-18}$ jetting, ${ }^{19}$ and atomization ${ }^{20}$ ) and manipulate particles (handling, ${ }^{21}$ focusing, ${ }^{22}$ separation, ${ }^{23,24}$ sorting, ${ }^{25,26}$ concentration, ${ }^{27-30}$ and reorientation $^{31}$ ) in lab-on-a-chip devices for applications in chemistry, biology, and medicine. The ability for a SAW to mix, translate, jet, and atomize droplets is dictated by its amplitude. For small amplitudes, fluid mixing is favored; for intermediate amplitudes the droplet is translated, and for higher amplitudes one can observe fluid jetting and atomization. Other systems that call for quantitative SAW analyses are quantum systems such as quantum dots, ${ }^{32-35}$ photonic cavities, ${ }^{36-38}$ single carrier transport systems, ${ }^{39-41}$ and SAW driven single electron transport (SAW/SET) in two dimensional

${ }^{\text {a)} E l e c t r o n i c ~ m a i l: ~ i b r a h i m a . s . c a m a r a @ g m a i l . c o m ~}$ electron gas (2DEG) heterostructures ${ }^{42,43}$ like $\mathrm{GaAs} / \mathrm{Al}_{x} \mathrm{Ga}_{1-x}$ As. In 2DEG heterostructures, the SAWs can induce a quantized current $I=n e f$, where $n$ is an integer, $e$ is the elementary electron charge and $f$ is the frequency of the SAWs. For metrology applications this current needs to be generated with some accuracy. It was demonstrated that the accuracy of the quantized current can be enhanced by reducing the microwave heating. ${ }^{43}$ To achieve this goal, the interdigitated transducers must be optimized in order to reduce the RF voltage applied to the transducers without reducing the amplitude of the SAWs.

These active developments around the use of SAWs call for an easy method to quantify their amplitude. Finite element modeling (FEM) is a possible route for obtaining the amplitude of SAWs. ${ }^{4-49}$ But this method requires a high computational time and a powerful computer hardware ${ }^{49}$ for $3 \mathrm{D}$ simulations. Interferometric probes ${ }^{50}$ can be used as long as the laser spot focused on the surface is much smaller than the acoustic wavelength. Therefore, they are not useful for high frequency surface waves. Diffraction-based methods in the visible ${ }^{50,51}$ or X-ray domain ${ }^{52-56}$ can also give the amplitude of SAWs but require an optical access to the device under test. The need of an optical access makes these methods difficult to use in some environments like in cryogenic spaces for example. In some recent studies, the amplitude of Rayleigh waves excited by an IDT has been estimated by an electrical method. ${ }^{38,57,58}$ This method is based on S-parameter $\mathrm{S}_{i j}$ measurements of the SAW device by a Vector Network Analyzer (VNA) as a function of the microwave frequency. The magnitude of the reflection coefficients $S_{i i}$ exhibits a dip at the frequency $f_{0}$ of emission of the acoustic waves. Without considering the effects of the fixtures, the acoustic reflections between the IDT fingers and electrode sheet resistance, the electric power converted into acoustic power is then estimated by $\mathcal{P}_{a}=\Delta\left|S_{i i}\right|^{2} \mathcal{P}_{\text {rf }}$, where $\mathcal{P}_{\text {rf }}$ is the 
RF power applied to the IDT and $\Delta\left|S_{i i}\right|^{2}$ is the amplitude of the dip. After that, the amplitude of the SAW is calculated by solving the equation $\mathcal{P}_{a}=2 \mathcal{P}_{\text {SAW }}$ where the factor 2 accounts for the bidirectional emission of two waves, and $\mathcal{P}_{\text {SAW }}$ is the total acoustic power flow across the acoustic beam and is given by ${ }^{50}$

$$
\mathcal{P}_{\mathrm{SAW}}=2 W \rho v_{R} \pi^{2} f_{0}^{2} \int_{0}^{\infty}\|\vec{u}(x, z)\|^{2} d z
$$

where $\rho, W, v_{R}$, and $\vec{u}(x, y, z)$ are, respectively, the density of the substrate, the aperture of the transducer, the velocity, and the particle displacement vector of the Rayleigh wave in a reference frame associated to the substrate. This method based only on the $\left|S_{i i}\right|^{2}$ dip, has no theoretical grounds, and we will show that it underestimates the displacement when electric losses in the transducer are not negligible. In this manuscript another strategy is considered to calculate the amplitude of the displacements from S-parameter measurements. By using the Coupling-of-Modes (COM) model and the P-Matrix formalism, a complete characterization of the SAW device and the fixtures is performed in order to include the effects of the peripheral circuit, the IDT electrode resistance, the acoustic attenuation beneath the transducers and the acoustic reflection due to the thin metal electrodes. The obtained displacements are then compared to the acoustic displacement measured directly by X-ray diffraction. The COM model was recently implemented to optimize the IDT design for minimum microwave heating. ${ }^{43}$ Here we use it to extract the acoustic wave induced displacement, which we compare to those measured directly by X-ray diffraction.

\section{SAMPLE}

A surface acoustic wave filter, whose schematic is shown in Fig. 1(a), was deposited on the (001) Gallium Arsenide (GaAs) surface. It is composed of two identical interdigitated transducers (IDTs) separated by $l_{\text {gap }}=3 \mathrm{~mm}$. The period of the IDT fingers is $\lambda=5.2 \mu \mathrm{m}$, the metallization ratio, 0.5 , the aperture, $W=2 \mathrm{~mm}$ and the number of IDT finger pairs, $N=60$. The electrodes are made of $\mathrm{Cr} / \mathrm{Au}$ of nominal thickness $10 / 100 \mathrm{~nm}$. The excitation signal is brought to the interdigitated transducers by means of coaxial cables and coplanar waveguides of impedance $50 \pm 2 \Omega$ and straight round bond-wires. The coaxial cables have an approximate length of $l_{\text {coax }}=10 \mathrm{~cm}$ and a nominal attenuation around $1 \mathrm{~dB} / \mathrm{m}$ between $400 \mathrm{MHz}$ and $1000 \mathrm{MHz}$. The bond wires are made of copper and have an approximate total length $l_{\text {wire }}=9 \mathrm{~mm}$ for each transducer and a diameter $d_{\text {wire }}=30 \mu \mathrm{m}$. The self-inductance associated to these wires is then theoretically estimated to be ${ }^{59} L_{\text {wire }}=200$ $\times l_{\text {wire }}\left[\ln \left(4 l_{\text {wire }} / d_{\text {wire }}\right)-0.75\right]=11.4 \mathrm{nH}$. The scattering twoport S-parameters of the system were measured by the Vector Network Analyzer after an accurate SOLT (ShortOpen-Load-Thru) calibration between $0.1 \mathrm{MHz}$ and $1 \mathrm{GHz}$.

Using the elastic constants $C_{11}=118.4 \mathrm{GPa}, C_{12}=53.7$ $\mathrm{GPa}, C_{44}=59.1 \mathrm{GPa}$ and the density $\rho=5317 \mathrm{~kg} / \mathrm{m}^{3}$ of Gallium Arsenide given by Cottam et al. ${ }^{60}$ the allowed SAW mode propagating on the free boundary of a semi-infinite
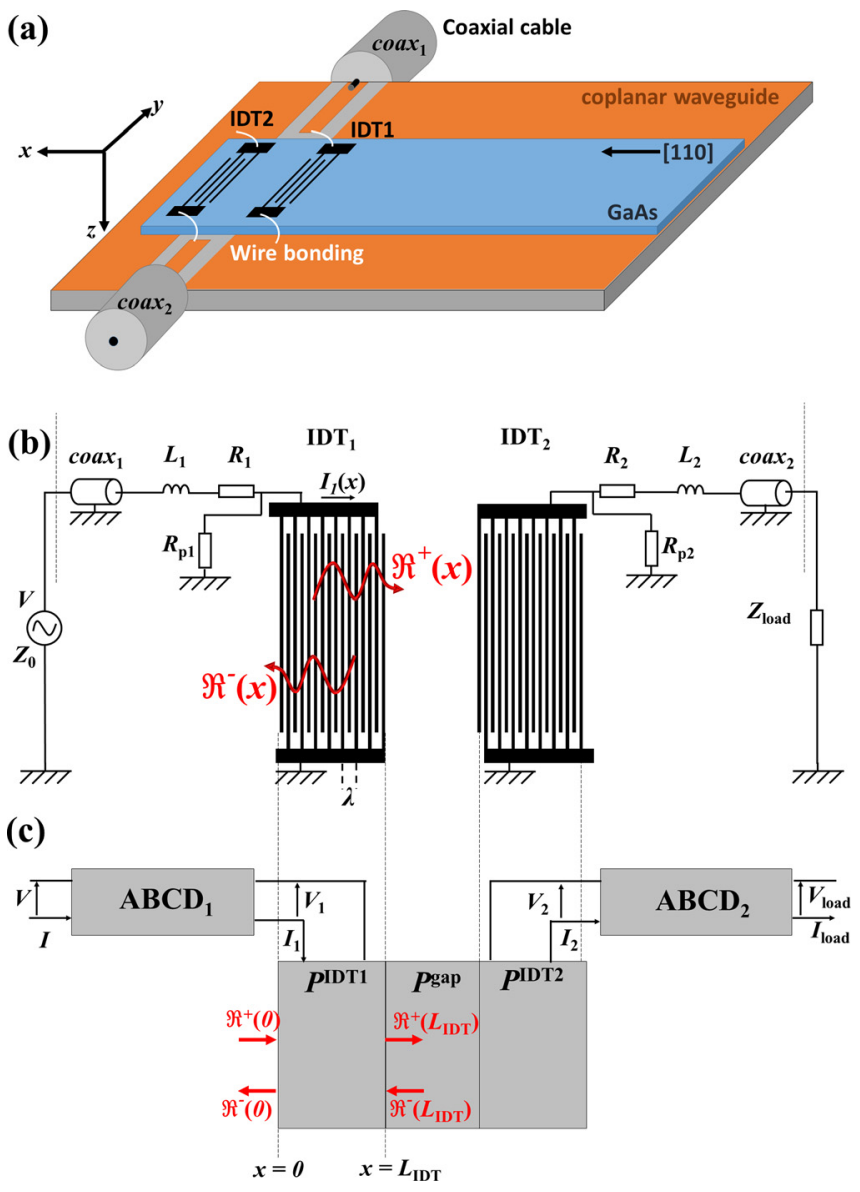

FIG. 1. (a) Schematic and (b) equivalent electrical circuit of the SAW filter and the fixtures. The fixtures are composed of coaxial lines $\left(\operatorname{coax}_{1}\right.$ and $\operatorname{coax}_{2}$ ), the self-inductances $L_{1}$ and $L_{2}$ of the wires, the electrical series resistances $R_{1}$ and $R_{2}$ and shunt resistances $R_{p, 1}$ and $R_{p, 2}$ of the transducers. $\Re^{+}(x)$ and $\Re^{-}(x)$ are the surface acoustic wave amplitudes for the forward and backward directions, respectively. They are defined such that the associated SAW powers are $\left|\Re^{+}(x)\right|^{2}$ and $\left|\Re^{-}(x)\right|^{2}$ at the point of coordinate $x$. (c) The coaxial lines, inductances, and resistances are described by transfer matrices $\mathrm{ABCD}_{1}$ and $\mathrm{ABCD}_{2}$, the interdigitated transducers are represented by P-matrices $P_{1}$ and $P_{2}$, and the gap between the transducers is represented by the P-matrix $P_{\text {gap }}$.

half-space of (001)-cut GaAs was obtained by solving the elastic wave equation. ${ }^{61}$ This acoustic mode is of Rayleigh type and the transducers excite the waves that propagate along the [110] direction of GaAs with a theoretical Rayleigh velocity of $v_{R}=2853 \mathrm{~m} \mathrm{~s}^{-1}$ at $300 \mathrm{~K}$, corresponding to a resonance frequency $f_{0}=v_{R} / \lambda=548 \mathrm{MHz}$. The particle displacement vector $\vec{u}$ associated to the Rayleigh acoustic mode in GaAs, propagating towards $x>0$, is given by

$$
\begin{aligned}
\frac{\vec{u}(x, z)}{U} & =\vec{u}_{n}(x, z) \\
& =2 \mathrm{e}^{-q_{r} k_{R} z}\left[\begin{array}{c}
\cos \left(q_{i} k_{R} z+\varphi / 2\right) \cos \left(\omega t-k_{R} x\right) \\
0 \\
-r \sin \left(q_{i} k_{R} z-\psi+\varphi / 2\right) \sin \left(\omega t-k_{R} x\right)
\end{array}\right]
\end{aligned}
$$

in the reference frame $(x, y, z)$ indicated in Fig. 1(a), where $k_{R}=2 \pi / \lambda$ and $q_{r}, q_{i}, r, \varphi$ and $\psi$ are functions of the elastic constants of GaAs. Their values are $q_{r}=0.5, q_{i}=-0.48$, 
$r=1.34, \varphi=-2.10 \mathrm{rd}$, and $\psi=2.61 \mathrm{rd}$ at $300 \mathrm{~K}$ and for a propagation along [110]. The displacement components $u_{x}$ and $u_{z}$, along $x$ and $z$, respectively, decay with depth below the free surface and vanish within a distance of the order of the wavelength. Since all the parameters in the right hand side of Eq. (2) are known, the amplitude of the SAWs is only related to the value of $U$ which depends on the characteristics of the interdigitated transducers (aperture, type of metal, and metallization ratio), the peripheral circuits (damping in cables, matching circuit, parasitic resistance, inductance, and capacitance.) and incident RF power.

\section{THE COUPLING-OF-MODES MODEL}

\section{A. Description}

The Coupling-of-Modes is a phenomenological model for guided wave excitation and propagation, which is widely employed for the design of high-performance SAW devices. ${ }^{62-66}$ This model has the advantage of including the effects of propagation loss beneath the transducer, electrode reflections, electrical transduction, and distributed finger capacitance. Let us consider the transducer IDT $_{1}$ schematically represented in Fig. 1(b). Like Wu et al. ${ }^{67}$ we assume here that the effects of the electrode resistance of the real transducer can be accounted by simply putting a resistance $R_{1}$ in series with the impedance of an ideal transducer. The value of $R_{1}$ can be estimated ${ }^{68} R_{1} \simeq 8 \rho_{\mathrm{Au}} W / N h_{e} \lambda=12.5 \Omega$, where $\rho_{\mathrm{Au}}=2.2 \times 10^{-8} \Omega \mathrm{m}$ is the resistivity of gold at $300 \mathrm{~K}$ (Ref. 69) and $h_{e}$ is the electrode metal thickness. For an ideal transducer, the DC resistance $R_{p}$ between the contact pads must be infinite (no electrical contacts between finger electrodes connected to different contact pads and no conductive substrate). For our transducer $\mathrm{IDT}_{1}$, the measured DC resistance is $R_{p, 1}=879 \Omega$ and its effect is accounted for by adding a shunt resistance of value $R_{p, 1}$ in the circuit model shown in Fig. 1(b).

For an incident RF signal of power $\mathcal{P}_{\text {rf }}$, let $V_{1}$ be the voltage applied to the transducer IDT $_{1}$ (see Figure 1(c)). The COM equations that govern the SAW mode amplitudes $\Re^{+}(x, f)$ and $\Re^{-}(x, f)$ generated by the transducer IDT $_{1}$ and propagating in the $\pm x$ directions, respectively, (see Figure 1(b)) are

$$
\begin{gathered}
\frac{\mathrm{d} \Re^{+}(x)}{\mathrm{d} x}=-i \delta \Re^{+}(x)+i \kappa \Re^{-}(x)+i \alpha V_{1}, \\
\frac{\mathrm{d} \Re^{-}(x)}{\mathrm{d} x}=-i \kappa^{*} \Re^{+}(x)+i \delta \Re^{-}(x)-i \alpha^{*} V_{1},
\end{gathered}
$$

and

$$
\frac{\mathrm{d} I_{1}(x)}{\mathrm{d} x}=-2 i \alpha^{*} \Re^{+}(x)-2 i \alpha \Re^{-}(x)+i 2 \pi f C_{1} V_{1},
$$

where $I_{1}$ is the busbar current, $C_{1}$ is the static capacitance of the transducer per unit length, $\delta=2 \pi\left(f-f_{0}\right) / v-i \gamma$ is the detuning parameter, and $\gamma$ is the attenuation of the acoustic waves. For a metallization ratio of $0.5, C_{1}$ can be theoretically approximated ${ }^{50,66}$ as $C_{1} \simeq \varepsilon_{0}\left(\varepsilon_{r}+1\right) W / \lambda_{0}=44 \mathrm{fF} / \mu \mathrm{m}$ where $\varepsilon_{0}$ is the permittivity of free space and $\varepsilon_{r} \simeq 12$ is the relative permittivity of GaAs. ${ }^{70}$ In Equations (3)-(5) the COM parameters that have to be determined are the reflectivity $\kappa$, the transduction coefficient $\alpha$, the attenuation $\gamma$, and the acoustic velocity $v$ beneath the transducer. Since the $\mathrm{COM}$ equations are linear, the solutions are usually presented in the P-matrix form

$$
\left[\begin{array}{c}
\Re^{-}(0) \\
\Re^{+}\left(L_{i d t}\right) \\
I_{1}
\end{array}\right]=\left[\begin{array}{lll}
P_{11} & P_{12} & P_{13} \\
P_{21} & P_{22} & P_{23} \\
P_{31} & P_{32} & P_{33}
\end{array}\right]\left[\begin{array}{c}
\Re^{+}(0) \\
\Re^{-}\left(L_{i d t}\right) \\
V_{1}
\end{array}\right],
$$

where $L_{i d t}=N \lambda$ is the length of the transducer IDT ${ }_{1}$, and the components $P_{i j}$ of the P-matrix of $\mathrm{IDT}_{1}$ are given by Plessky et al. ${ }^{66}$ as functions of the COM parameters. A system of equations similar to the one given in Eq. (6) can be derived to describe the waves propagating over the gap between the transducers and over the transducer $\mathrm{IDT}_{2}$ yielding the $\mathrm{P}$ matrices $P^{\text {gap }}$ and $P^{\mathrm{IDT}_{2}}$ of the gap and the transducer $\mathrm{IDT}_{2}$ (see Fig. 1(c)). The components of the P-matrix of the gap are $P_{j j}^{g a p}=P_{j 3}^{g a p}=P_{3 j}^{g a p}=0(j=1,2$, or 3$)$ and $P_{12}^{\text {gap }}=P_{21}^{\text {gap }}$ $=\exp \left(-i \times l_{\text {gap }}\left[2 \pi f / v_{R}-i \gamma_{\text {gap }}\right]\right)$.

\section{B. Determination of the COM parameters}

To recover the values of the COM parameters, the method reported by Wu et al ${ }^{67}$ for a one-port SAW resonator was adapted to our problem. Their method is based on the derivation of the theoretical admittance $Y^{\text {theo }}$ of a oneport resonator from the P-matrix and the comparison of this derived admittance to the measured admittance $Y^{\mathrm{IDT}}$ of the transducer. Using an optimization algorithm, they determined the COM parameters that minimize the error function $e=\sum_{n=1}^{N_{\mathrm{f}}}\left|Y^{\mathrm{IDT}}(n)-Y^{\text {theo }}(n)\right|^{2}$, where $N_{\mathrm{f}}$ is the number of data points.

Before the minimization process of the error function $e$, the admittances $Y_{11}^{\mathrm{IDT}}$ and $Y_{22}^{\mathrm{IDT}}$ of the SAW filter, i.e., without the contribution of the fixtures, need to be deduced from the measured S-parameters. Figure 2(a) shows the reflection parameter $S_{11}$ of the SAW device including the contribution of the fixtures. To obtain the admittance of the transducer $\operatorname{IDT}_{j}(j=1$ or 2$)$, the electrical contributions of the fixtures, i.e., the length $l_{\text {coax }, j}$ of the coaxial cable, the self-inductance $L_{j}$ of the bond-wires, the electrode resistance $R_{j}$, and the shunt resistance $R_{p, j}$ need to be quantified and removed. The electrical characteristics of the fixtures were obtained by fitting separately the measured reflections $S_{11}$ and $S_{22}$ of IDT 1 and $\mathrm{IDT}_{2}$, respectively, to

$$
S_{j j}=\frac{Z_{j}-Z_{0}}{Z_{j}+Z_{0}} \mathrm{e}^{-i 2 \theta_{j}}
$$

where

$$
Z_{j}=i L_{j} \omega+R_{j}+\frac{R_{p, j}}{1+i R_{p, j} C_{j} L_{i d t} \omega},
$$

$Z_{0}=50 \Omega, \theta_{j}=\omega l_{\text {coax } j} \sqrt{\varepsilon_{t}} / c, \omega=2 \pi f, \varepsilon_{t}=2.1$ is the relative permittivity of teflon, $c$ is the celerity of light in free space, and the subscript $j$ refers to the transducers $(j=1$ for 


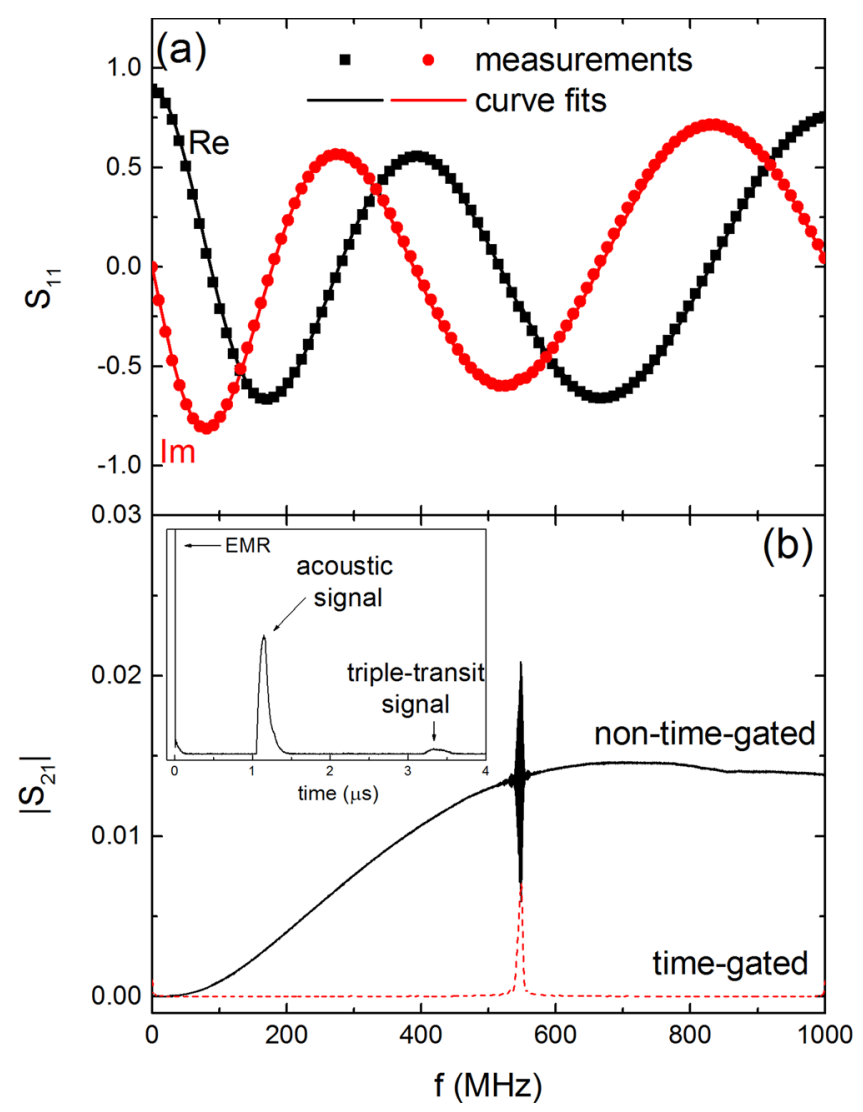

FIG. 2. (a) Comparison between measured (dots) and fitted (solid lines) reflection coefficients $S_{11}$. (b) non-time-gated and time-gated measured amplitude of $S_{21}$. The non-zero baseline exhibited by the non-time-gated measurements is due to the electromagnetic radiations (EMR) between the two interdigitated transducers. Inset: Amplitude of $S_{21}$ in time domain showing the EMR, the acoustic signal, and the triple-transit signal.

$\mathrm{IDT}_{1}$ and $j=2$ for $\mathrm{IDT}_{2}$ ). Equation (8) is obtained by considering that the transducer IDT $_{j}$ is a simple capacitor of capacitance $C_{j} L_{i d t}$. Figure 2(a) shows the curve fit results of $S_{11}$ between 0.1 and $1000 \mathrm{MHz}$ using the values $l_{\text {coax }, 1}=12.37$ $\mathrm{cm}, L_{1}=12.0 \mathrm{nH}, R_{1}=11.75 \Omega, C_{1}=55.1 \mathrm{fF} / \mu \mathrm{m}$. A similar curve fit of $S_{22}$ for IDT IDields $_{2}$ coax $, 2=12.43 \mathrm{~cm}, L_{2}=$ $9.46 \mathrm{nH}, R_{2}=15.41 \Omega, C_{2}=53.6 \mathrm{fF} / \mu \mathrm{m}$. Note that the values of resistance, inductance, and capacitance obtained from the curve fits are quite similar to the values estimated previously from the theoretical expressions.

Besides the reflection coefficients $S_{11}$ and $S_{22}$, the transmission coefficients $S_{21}$ and $S_{12}$ were also measured. The amplitude of $S_{21}$ exhibits a peak at $548 \mathrm{MHz}$, as shown by the full lines in Fig. 2(b). This peak is due to the emission (at $\mathrm{IDT}_{1}$ ) and detection (by $\mathrm{IDT}_{2}$ ) of an acoustic wave. The amplitude of $S_{21}$ reveals also a non-zero baseline between $0.1 \mathrm{MHz}$ and $1000 \mathrm{MHz}$ due to the electromagnetic radiations (EMR) between the two transducers. ${ }^{71}$ These EMR travel at the speed of light and are detected by IDT $_{2}$ (if emitted by IDT $_{1}$ ) with a delay of $1 \mu$ s before the acoustic signal is detected (see inset of Fig. 2(b)). By taking profit of the time-gating capability of the vector network analyzer, the EMR signal was mathematically filtered out in the time domain, eliminating then the non-zero baseline of $S_{21}$ in the frequency domain (see time-gated curve in Fig. 2(b)). In the following, the calculations are done using the time-gated form of the transmission parameters $S_{21}$ and $S_{12}$.

After the contributions of the fixtures are removed from the S-parameters matrix by de-embedding, the admittance matrix $Y^{\mathrm{IDT}}$ of the SAW device (without the contribution of the fixtures) is deduced using the relationship between admittance and S-parameters. ${ }^{72}$ Finally, a Matlab nonlinear optimization algorithm is used to minimize the error function $e_{i j}=\sum_{n=1}^{N_{\mathrm{f}}}\left|Y_{i j}^{\mathrm{IDT}}(n)-Y_{i j}^{\text {theo }}(n)\right|^{2}$ in order to determine the COM parameters $v, \kappa, \alpha$, and $\gamma$. To avoid non-physical local minima during the minimization process, an initial guess of the COM parameters needs to be done. In the following, the reflectivity $\kappa$, the transduction $\alpha$, and the attenuation $\gamma$ will be given in their normalized form $\kappa^{\prime}=-\kappa \lambda, \alpha^{\prime}=\alpha \lambda$, and $\gamma^{\prime}=\gamma \lambda$. For the initial guess of the acoustic velocity $v$ beneath the transducers, the slowing of the wave due to metal loading can be accounted for by FEM modeling ${ }^{67}$ or by using a formula ${ }^{66}$ based on the perturbation theory and giving the relative variation of the velocity as function of the electrode thickness and the electromechanical coupling constant $K$. Since the electromechanical coupling of GaAs is small $\left(K^{2}=0.07 \%\right.$ (Ref. 50) ) and the electrode thickness of our transducers is negligible compared to the wavelength $\lambda$, we choose the theoretical Rayleigh wave velocity $v_{\mathrm{R}}=2853$ $\mathrm{m} / \mathrm{s}$, calculated for the free surface of GaAs, as an initial guess for the velocity $v$. The initial estimations of the transduction $\alpha^{\prime}$ and the reflectivity $\kappa^{\prime}$ are given by ${ }^{66,67} \alpha^{\prime} \simeq 3$ $\sqrt{v_{\mathrm{R}} W K^{2} \varepsilon_{0} \varepsilon_{r} / 2 \lambda_{0}}$ and $\kappa^{\prime} \simeq-2\left[R_{e} K^{2} / 2+R_{m} h_{e} / \lambda_{0}\right]$ where $R_{e}=-0.7178$ (for a metallization ratio of 0.5 ) and $R_{m}$ $=-0.0242$ (for $\mathrm{GaAs}^{65}$ ) are the electrical and mechanical contributions, respectively. The initial estimations and the recovered COM parameters after the minimization process are listed in Table I. The full curves in Figs. 3(a) and 3(b) are the admittances obtained after the minimization process. From the comparison between the measured and calculated transmission admittance coefficient $Y_{21}$ (or $Y_{12}$ ) between $\mathrm{IDT}_{1}$ and $\mathrm{IDT}_{2}$ we obtain the experimental unperturbed Rayleigh wave velocity $v_{\mathrm{R}}=2860.5 \mathrm{~m} / \mathrm{s}$ on the free surface of GaAs.

\section{SURFACE ACOUSTIC WAVE AMPLITUDE}

In the following, we calculate the total acoustic power $\mathcal{P}_{a}$ carried by the counter-propagating waves $\Re^{+}(x)$ and $\Re^{-}(x)$ at the point of coordinate $L_{\text {idt }}$ between the transducers (near the transducer $\left.\operatorname{IDT}_{1}\right)$. The waves $\Re^{+}(x)$ and $\Re^{-}(x)$ may be interpreted as stresses, displacements, or electrical potentials, but usually they are normalized so that the

TABLE I. Guessed and fitting COM parameters from the admittance coefficients $Y_{11}^{\mathrm{IDT}}, Y_{22}^{\mathrm{IDT}}$, and $Y_{21}^{\mathrm{IDT}}$.

\begin{tabular}{lcccc}
\hline \hline COM parameters & Initial guess & IDT 1 & IDT 2 & Gap \\
\hline$v(\mathrm{~m} / \mathrm{s})$ & 2853 & 2847 & 2851 & $2860.5^{\mathrm{a}}$ \\
$\kappa^{\prime}(\%)$ & 0.14 & 0.89 & 0.73 & \\
$\alpha^{\prime}\left(10^{-4} / \sqrt{\Omega}\right)$ & 6 & 6.21 & 6.24 & \\
$\gamma^{\prime}\left(10^{-4} \mathrm{~Np} / \lambda_{0}\right)$ & 3.6 & 20 & 18 & 14 \\
\hline \hline
\end{tabular}

${ }^{\mathrm{a}}$ Measured Rayleigh velocity $v_{\mathrm{R}}$ on the free surface of GaAs. 


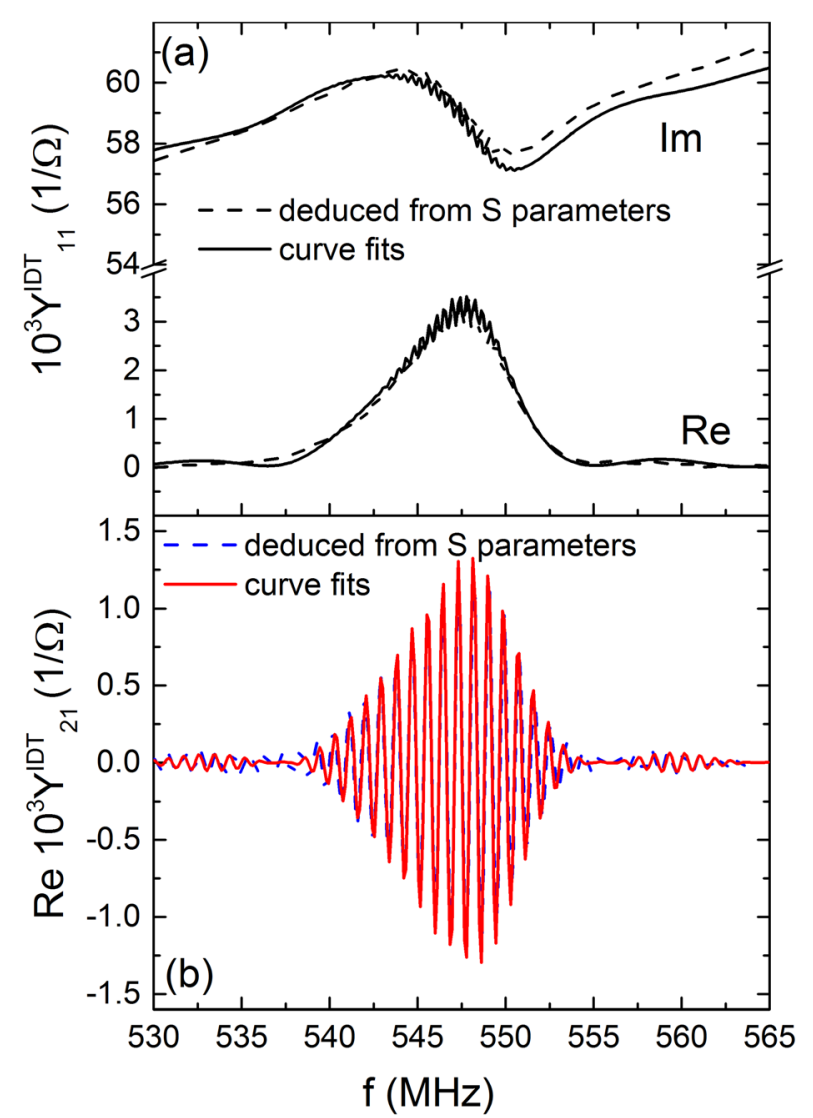

FIG. 3. Comparison between de-embedded (dashed lines) and fitted (solid lines) admittance coefficients $Y_{11}^{\mathrm{IDT}}$ (a) and $Y_{21}^{\mathrm{IDT}}$ (b) of the SAW filter.

acoustic power associated to each wave is given by ${ }^{50}$ $\left|\Re^{ \pm}\left(L_{i d t}\right)\right|^{2}$. The amplitudes of $\Re^{+}\left(L_{\text {idt }}\right)$ and $\Re^{-}\left(L_{\text {idt }}\right)$ can be obtained from Eq. (6) by assuming that $\Re^{+}(0)=0$ (no waves coming from outside the SAW filter). They are given by $\Re^{-}\left(L_{\text {idt }}\right)=\left(I_{1}-P_{33} V_{1}\right) / P_{32}$ and $\Re^{+}\left(L_{\text {idt }}\right)=P_{22} \Re^{-}$ $+P_{23} V_{1}$, where the applied voltage $V_{1}$ and the current $I_{1}$ (see Fig. 1) are related to the incident RF power $\mathcal{P}_{\text {rf }}$, the length of the coaxial cable, the inductance $L_{1}$ and the resistances $R_{1}$ and $R_{p, 1}$ by (see the Appendix)

$$
\begin{aligned}
V_{1}= & \frac{V}{Z_{0}}\left[Z_{0} \cos \theta_{1}+i\left(R_{1}+i L_{1} \omega\right) \sin \theta_{1}\right] \\
& -i I\left[Z_{0} \sin \theta_{1}-i\left(R_{1}+i L_{1} \omega\right) \cos \theta_{1}\right]
\end{aligned}
$$

and

$$
\begin{aligned}
I_{1}= & I\left[\frac{R_{p, 1}+R_{1}+i L_{1} \omega}{R_{p, 1}} \cos \theta_{1}+i \frac{Z_{0}}{R_{p, 1}} \sin \theta_{1}\right] \\
& -i \frac{V}{Z_{0}}\left[\frac{R_{p, 1}+R_{1}+i L_{1} \omega}{R_{p, 1}} \sin \theta_{1}-i \frac{Z_{0}}{R_{p, 1}} \cos \theta_{1}\right]
\end{aligned}
$$

where $^{73} V=\sqrt{\mathcal{P}_{\text {rf }} Z_{0}}\left(1+S_{11}\right), I=\sqrt{\mathcal{P}_{\text {rf }} / Z_{0}}\left(1-S_{11}\right)$ and $S_{11}$ can be either calculated using Eq. (7) or measured directly with a VNA. The value of $\Re^{+}\left(L_{\text {idt }}\right)$ at the resonance frequency $f_{0}$ was found to be over 10 times higher than the value of $\Re^{-}\left(L_{\text {idt }}\right)$ as expected since $\Re^{-}\left(L_{\text {idt }}\right)$ is due to the acoustic signal reflected from $\operatorname{IDT}_{2}$. Neglecting $\Re^{-}\left(L_{\text {idt }}\right)$, the total acoustic power delivered by our bidirectional transducer $\operatorname{IDT}_{1}$ is $\mathcal{P}_{a}=2\left|\Re^{+}\left(L_{\mathrm{idt}}\right)\right|^{2}=2\left|P_{23} V_{1}\right|^{2}$. From the latter relation, Eqs. (2) and (1), we derive the expression of the SAW amplitude $U$

$$
U=\left|P_{23} V_{1}\right| \frac{\mathcal{F}\left(r, q_{r}, q_{i}, \varphi, \psi\right)}{\sqrt{\pi f_{0} \rho v_{R}^{2} W}},
$$

where $V_{1}$ is given by Eq. (9), $P_{23}$ is the voltage-to-SAW transduction factor of the transducer $\mathrm{IDT}_{1}$ and the dimensionless factor $\mathcal{F}=1 / \sqrt{k_{R} \int_{0}^{\infty}\left\|\vec{u}_{n}\right\|^{2} d z}$ depends only on the elastic constants of GaAs. $P_{23}$ and $\mathcal{F}$ are, respectively, given by

$$
\begin{aligned}
P_{23}= & (-1)^{N+1} \alpha L_{i d t} \frac{\sin \left(q L_{i d t} / 2\right)}{q L_{i d t} / 2} \\
& \times \frac{(\delta+\kappa) \sin \left(q L_{i d t} / 2\right)-i q \cos \left(q L_{i d t} / 2\right)}{q \cos q L_{i d t}+i \delta \sin \left(q L_{i d t}\right)},
\end{aligned}
$$

for a bidirectional uniform transducer, ${ }^{66}$ and

$$
\mathcal{F}=\sqrt{\frac{q_{r}\left(q_{r}^{2}+q_{i}^{2}\right)}{q_{r}\left[q_{r} \cos (\varphi)-q_{i} \sin (\varphi)\right]+\left(1+r^{2}\right)\left(q_{i}^{2}+q_{r}^{2}\right)+r^{2} q_{r}\left[q_{i} \sin (-2 \psi+\varphi)-q_{r} \cos (-2 \psi+\varphi)\right]}},
$$

where $q=\sqrt{\delta^{2}-\kappa^{2}}$.

For a Rayleigh wave propagating along the [110] direction of GaAs at $300 \mathrm{~K}$ the calculated value of $\mathcal{F}$ is 0.46 . Using the values of the COM parameters obtained for IDT $_{1}$ (Table I) we calculate the transduction factor $P_{23}$ $=0.033 \Omega^{-0.5}$ at $f=f_{0}$. From the COM model, the variation of $U$ with respect to the incident $\mathrm{RF}$ power $\mathcal{P}_{\text {rf }}$ is $U_{\mathrm{COM}}$ $=1.45 \sqrt{\mathcal{P}_{\text {rf }}}$ (see full lines in Fig. 5) where $U_{\mathrm{COM}}$ is given in Angström and $\mathcal{P}_{\text {rf }}$ in watts. This is the main result of this approach, from which the SAW amplitude can then be computed for any injected RF power, in the linear regime. The displacement was also estimated from the dip in $\left|S_{11}\right|^{2}$ (see Figure 4) as $\mathcal{P}_{a}=\Delta\left|S_{11}\right|^{2} \mathcal{P}_{\mathrm{rf}}$, which is the method commonly used. ${ }^{38,57,58}$ From the amplitude $\Delta\left|S_{11}\right|^{2} \approx 0.02$ of the dip, the variation of $U$ with respect to the incident RF power $\mathcal{P}_{\text {rf }}$ is $U_{\text {dip }}=1.18 \sqrt{\mathcal{P}_{\text {rf }}}$ (see dotted lines in Fig. 5). Finally, the amplitude $U$ was also measured using $\mathrm{X}$-ray diffraction around the (002) Bragg peaks of the GaAs substrate. The details of the X-ray studies are reported elsewhere. ${ }^{55}$ The value of $U$ was measured up to $\mathcal{P}_{\text {rf }}=320 \mathrm{~mW}$ and the variation of $U_{\mathrm{XRD}}=1.46 \sqrt{\mathcal{P}_{\mathrm{rf}}}$ (see dashed lines in Fig. 5) was obtained by performing a linear fit of the data points. The 


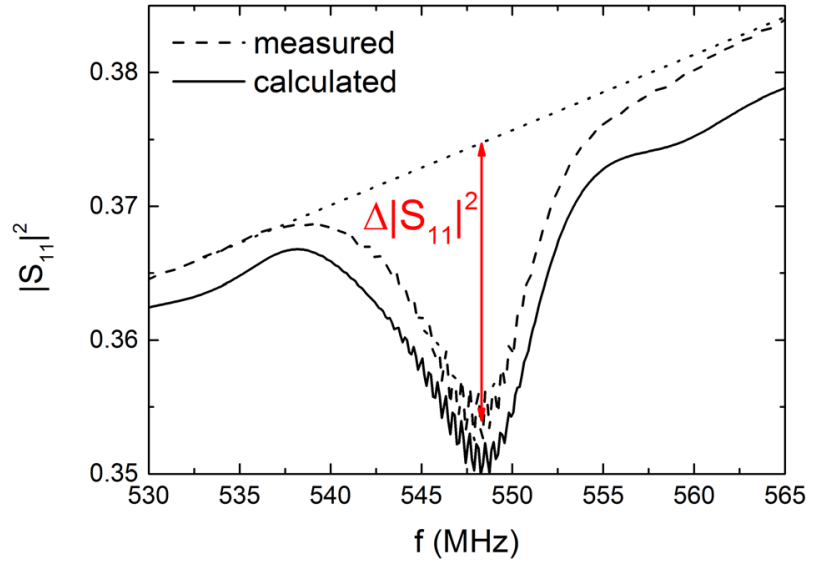

FIG. 4. Comparison between measured (dashed line) and calculated (solid line) square of the reflection coefficient $\left|S_{11}\right|^{2} . \Delta\left|S_{11}\right|^{2}$ can only provide a rough estimate of the wave amplitude (see main text and Fig. 5).

variation of $U$ obtained by X-ray diffraction is in perfect agreement with the variation obtained by our analysis using the COM model whereas, the more simple approach based on $\Delta\left|S_{11}\right|_{\text {dip }}^{2}$ underestimates it by a factor of 1.24 because of the electrical losses (electrical resistance of the transducer). For some applications, an increase of the amplitude by a factor of 1.24 can produce non-negligible effects. For example, it has been reported recently ${ }^{10}$ that for the magnetostrictive and ferromagnetic material $(\mathrm{Ga}, \mathrm{Mn})(\mathrm{As}, \mathrm{P})$, an increase of the surface acoustic wave strain $\epsilon_{i j}=0.5 \cdot\left(\delta u_{i} / \delta j+\delta u_{j} / \delta i\right)$ (the strain is proportional to the amplitude) by a factor of 1.2 can reduce the coercive field to about $1 \mathrm{mT}$.

\section{CONCLUSION}

We have presented an electrical method to measure the amplitude of surface acoustic waves generated by an interdigitated transducer and propagating on the free surface of a semi-infinite half-space of (001)-cut Gallium Arsenide. The method is based on the Vector Network Analyzer measurements of the S-parameters of the transducers and their

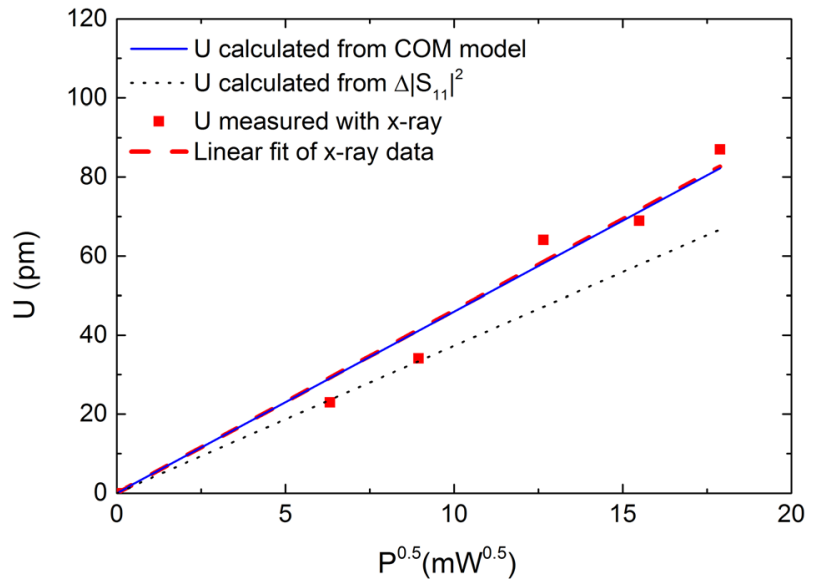

FIG. 5. Comparison between the displacements $U$ calculated from the COM model (full line) and from the dip $\left|\Delta S_{11}\right|^{2}$ (dotted line) as a function of the square root of the input RF power. The values of $U$ measured with X-ray diffraction are indicated by the square dots. The linear fit of the X-ray data (dashed line) is more in agreement with the values obtained from the COM model. modeling using a simple equivalent circuit and the Coupling-Of-Modes theory by taking into account the effects of the peripheral circuit (parasitic resistance, inductance, and capacitance.) and the resistance of the transducers. The amplitude of the surface acoustic waves obtained from our electrical analysis is in perfect agreement with the amplitude measured directly by X-ray diffraction showing that with careful modeling, displacements of a few angstroms can easily be evaluated electrically. This opens the way for an accurate and reliable determination of the SAW amplitude in magnetic systems, lab-on-a-chip devices, and quantum systems. Besides, unlike the diffraction-based methods which require an optical access to the device under test, the electrical method presented here does not necessitate an optical access and can thus be used when the device is in a windowless cryogenic space.

\section{ACKNOWLEDGMENTS}

We thank Christophe Rafaillac and Chris Lelong for the realization of a sample holder adapted to the RF cables, Loï Beccera for the realization of the IDTs and Catherine Gourdon and Jean-Yves Prieur for fruitful discussions and advice. This work has been supported by the French Agence Nationale de la Recherche (ANR13-JS04-0001-01).

\section{APPENDIX: RELATIONSHIP BETWEEN CURRENTS AND VOLTAGES}

In this Appendix, we explicitly give the derivation of Eqs. (9) and (10) which relate the input RF current $I$ and voltage $V$ applied to the coaxial cable $\operatorname{coax}_{1}$ to the output RF current $I_{1}$ and voltage $V_{1}$ that are seen by the transducer $\mathrm{IDT}_{1}$. The procedure is based on the ABCD transfer matrix formalism which expresses the input quantities in terms of the output quantities ${ }^{74}$

$$
\left[\begin{array}{l}
V \\
I
\end{array}\right]=\left[\begin{array}{ll}
A_{1} & B_{1} \\
C_{1} & D_{1}
\end{array}\right]\left[\begin{array}{l}
V_{1} \\
I_{1}
\end{array}\right],
$$

where $A_{1}, B_{1}, C_{1}$, and $D_{1}$ are the elements of the $\mathrm{ABCD}_{1}$ transfer matrix (see Fig. 1) of the microwave circuit network constituted by the transmission line $\operatorname{coax}_{1}$, the wire (modeled as a lumped inductor) $L_{1}$, the shunt resistance $R_{p, 1}$ and the series resistance $R_{1}$. The matrix $\mathrm{ABCD}_{1}$ is obtained from the transfer matrix of the individual components of the circuit thanks the cascaded network relation ${ }^{74}$

$$
\begin{aligned}
{\left[\begin{array}{ll}
A_{1} & B_{1} \\
C_{1} & D_{1}
\end{array}\right]=} & {\left[\begin{array}{ll}
A_{\text {coax } 1} & B_{\text {coax } 1} \\
C_{\text {coax } 1} & D_{\text {coax } 1}
\end{array}\right]\left[\begin{array}{cc}
A_{L 1} & B_{L 1} \\
C_{L 1} & D_{L 1}
\end{array}\right] } \\
& \times\left[\begin{array}{ll}
A_{R 1} & B_{R 1} \\
C_{R 1} & D_{R 1}
\end{array}\right]\left[\begin{array}{ll}
A_{R p 1} & B_{R p 1} \\
C_{R p 1} & D_{R p 1}
\end{array}\right],
\end{aligned}
$$

where

$$
\left[\begin{array}{cc}
A_{\text {coax } 1} & B_{\text {coax } 1} \\
C_{\text {coax } 1} & D_{\text {coax } 1}
\end{array}\right]=\left[\begin{array}{cc}
\cos (\theta) & i Z_{0} \sin (\theta) \\
\frac{i \sin (\theta)}{Z_{0}} & \cos (\theta)
\end{array}\right],
$$




$$
\begin{gathered}
{\left[\begin{array}{ll}
A_{L 1} & B_{L 1} \\
C_{L 1} & D_{L 1}
\end{array}\right]=\left[\begin{array}{cc}
1 & i L_{1} \omega \\
0 & 1
\end{array}\right],} \\
{\left[\begin{array}{ll}
A_{R 1} & B_{R 1} \\
C_{R 1} & D_{R 1}
\end{array}\right]=\left[\begin{array}{cc}
1 & R_{1} \\
0 & 1
\end{array}\right],}
\end{gathered}
$$

and

$$
\left[\begin{array}{ll}
A_{R p 1} & B_{R p 1} \\
C_{R p 1} & D_{R p 1}
\end{array}\right]=\left[\begin{array}{cc}
1 & 0 \\
\frac{1}{R_{p, 1}} & 1
\end{array}\right],
$$

are the transfer matrices of the transmission line, the wire, the shunt resistance $R_{p, 1}$ and the series resistance $R_{1}$, respectively. In Eq. (A3), $\theta$ is given by $\theta=2 \pi f_{0} l_{\text {coax }, 1} \sqrt{\varepsilon_{t}} / c$ where $\varepsilon_{t}=2.1$ is the relative permittivity of teflon. The losses in the coaxial cable $\operatorname{coax}_{1}$ can be accounted by adding an imaginary part to $\theta$. Once the expression of the matrix $\mathrm{ABCD}_{1}$ is obtained, it can be inverted, and the result can be multiplied by Eq. (A1) in order to obtain Eqs. (9) and (10).

${ }^{1}$ C. Ruppel, L. Reindl, and R. Weigel, IEEE Microwave Mag. 3, 65 (2002). ${ }^{2}$ A. Pohl, IEEE Trans. Ultrason., Ferroelectr. Freq. Control 47, 317 (2000).

${ }^{3}$ T. M. Gronewold, Anal. Chim. Acta 603, 119 (2007).

${ }^{4}$ K. Länge, B. E. Rapp, and M. Rapp, Anal. Bioanal. Chem. 391, 1509 (2008).

${ }^{5}$ J. Lee, Y.-S. Choi, Y. Lee, H. J. Lee, J. N. Lee, S. K. Kim, K. Y. Han, E. C. Cho, J. C. Park, and S. S. Lee, Anal. Chem. 83, 8629 (2011).

${ }^{6}$ L. Thevenard, J.-Y. Duquesne, E. Peronne, H. J. von Bardeleben, H. Jaffres, S. Ruttala, J.-M. George, A. Lemaitre, and C. Gourdon, Phys. Rev. B 87, 144402 (2013).

${ }^{7}$ L. Thevenard, I. S. Camara, S. Majrab, M. Bernard, P. Rovillain, A. Lemaitre, C. Gourdon, and J.-Y. Duquesne, Phys. Rev. B 93, 134430 (2016).

${ }^{8}$ W. Li, B. Buford, A. Jander, and P. Dhagat, IEEE Trans. Magn. 50, 37 (2014).

${ }^{9}$ W. Li, B. Buford, A. Jander, and P. Dhagat, J. Appl. Phys. 115, 17E307 (2014).

${ }^{10}$ L. Thevenard, I. S. Camara, J.-Y. Prieur, P. Rovillain, A. Lemaître, C. Gourdon, and J.-Y. Duquesne, Phys. Rev. B 93, 140405 (2016).

${ }^{11}$ J. Dean, M. T. Bryan, J. D. Cooper, A. Virbule, J. E. Cunningham, and T. J. Hayward, Appl. Phys. Lett. 107, 142405 (2015).

${ }^{12}$ S. S. P. Parkin, M. Hayashi, and L. Thomas, Science 320, 190 (2008),

${ }^{13}$ T. Frommelt, M. Kostur, M. Wenzel-Schäfer, P. Talkner, P. Hänggi, and A. Wixforth, Phys. Rev. Lett. 100, 034502 (2008).

${ }^{14}$ W.-K. Tseng, J.-L. Lin, W.-C. Sung, S.-H. Chen, and G.-B. Lee, J. Micromech. Microeng. 16, 539 (2006).

${ }^{15}$ T.-D. Luong, V.-N. Phan, and N.-T. Nguyen, Microfluid. Nanofluid. 10, 619 (2011).

${ }^{16}$ A. Wixforth, C. Strobl, C. Gauer, A. Toegl, J. Scriba, and Z. V. Guttenberg, Anal. Bioanal. Chem. 379, 982 (2004).

${ }^{17}$ A. Renaudin, P. Tabourier, V. Zhang, J. Camart, and C. Druon, Sens. Actuators, B 113, 389 (2006).

${ }^{18}$ T. A. Franke and A. Wixforth, ChemPhysChem 9, 2140 (2008).

${ }^{19}$ M. K. Tan, J. R. Friend, and L. Y. Yeo, Phys. Rev. Lett. 103, 024501 (2009).

${ }^{20}$ M. Kurosawa, T. Watanabe, A. Futami, and T. Higuchi, Sens. Actuators, A 50, 69 (1995).

${ }^{21}$ S. B. Q. Tran, P. Marmottant, and P. Thibault, Appl. Phys. Lett. 101, 114103 (2012).

${ }^{22}$ J. Shi, X. Mao, D. Ahmed, A. Colletti, and T. J. Huang, Lab Chip 8, 221 (2008).

${ }^{23}$ J. Shi, H. Huang, Z. Stratton, Y. Huang, and T. J. Huang, Lab Chip 9, 3354 (2009).

${ }^{24}$ J. Nam, Y. Lee, and S. Shin, Microfluid. Nanofluid. 11, 317 (2011).

${ }^{25}$ T. Franke, A. R. Abate, D. A. Weitz, and A. Wixforth, Lab Chip 9, 2625 (2009).

${ }^{26}$ T. Franke, S. Braunmüller, L. Schmid, A. Wixforth, and D. A. Weitz, Lab Chip 10, 789 (2010).
${ }^{27}$ R. Shilton, M. K. Tan, L. Y. Yeo, and J. R. Friend, J. Appl. Phys. 104, 014910 (2008).

${ }^{28} \mathrm{H}$. Li, J. R. Friend, and L. Y. Yeo, Biomed. Microdevices 9, 647 (2007).

${ }^{29}$ P. R. Rogers, J. R. Friend, and L. Y. Yeo, Lab Chip 10, 2979 (2010).

${ }^{30}$ M. K. Tan, J. R. Friend, and L. Y. Yeo, Lab Chip 7, 618 (2007).

${ }^{31}$ C. J. Strobl, C. Schaflein, U. Beierlein, J. Ebbecke, and A. Wixforth, Appl. Phys. Lett. 85, 1427 (2004).

${ }^{32}$ M. Metcalfe, S. M. Carr, A. Muller, G. S. Solomon, and J. Lawall, Phys. Rev. Lett. 105, 037401 (2010).

${ }^{33}$ M. Weiß, J. B. Kinzel, F. J. R. Schülein, M. Heigl, D. Rudolph, S. Morkötter, M. Döblinger, M. Bichler, G. Abstreiter, J. J. Finley, G. Koblmüller, A. Wixforth, and H. J. Krenner, Nano Lett. 14, 2256 (2014).

${ }^{34}$ F. J. R. Schülein, E. Zallo, P. Atkinson, O. G. Schmidt, R. Trotta, A. Rastelli, A. Wixforth, and H. J. Krenner, Nat. Nanotechnol. 10, 512 (2015).

${ }^{35}$ S. Lazić, E. Chernysheva, Ž. Gačević, H. P. van der Meulen, E. Calleja, and J. M. Calleja Pardo, AIP Adv. 5, 097217 (2015).

${ }^{36}$ M. M. de Lima, M. van der Poel, P. V. Santos, and J. M. Hvam, Phys. Rev. Lett. 97, 045501 (2006).

${ }^{37}$ D. A. Fuhrmann, S. M. Thon, H. Kim, D. Bouwmeester, P. M. Petroff, A. Wixforth, and H. J. Krenner, Nat. Photonics 5, 605 (2011).

${ }^{38} \mathrm{H}$. Li, S. A. Tadesse, Q. Liu, and M. Li, Optica 2, 826 (2015).

${ }^{39}$ P. V. Santos, F. Alsina, J. A. H. Stotz, R. Hey, S. Eshlaghi, and A. D. Wieck, Phys. Rev. B 69, 155318 (2004).

${ }^{40}$ A. García-Cristóbal, A. Cantarero, F. Alsina, and P. V. Santos, Phys. Rev. B 69, 205301 (2004).

${ }^{41}$ J. B. Kinzel, F. J. R. Schülein, M. Weiß, L. Janker, D. D. Bühler, M. Heigl, D. Rudolph, S. Morkötter, M. Döblinger, M. Bichler, G. Abstreiter, J. J. Finley, A. Wixforth, G. Koblmüller, and H. J. Krenner, ACS Nano 10, 4942 (2016)

${ }^{42}$ S. H. Simon, Phys. Rev. B 54, 13878 (1996).

${ }^{43}$ L. Song, H. Yuan, C. Zhang, L. Li, C. Lu, and J. Gao, J. Appl. Phys. 106, 104508 (2009).

${ }^{44}$ A. N. Darinskii, M. Weihnacht, and H. Schmidt, Lab Chip 16, 2701 (2016).

${ }^{45}$ L. Johansson, J. Enlund, S. Johansson, I. Katardjiev, and V. Yantchev, Biomed. Microdevices 14, 279 (2012).

${ }^{46}$ S. Yankin, A. Talbi, Y. Du, J.-C. Gerbedoen, V. Preobrazhensky, P. Pernod, and O. Bou Matar, J. Appl. Phys. 115, 244508 (2014).

${ }^{47}$ C. Gwiy-Sang and P. Duy-Thach, J. Korean Phys. Soc. 57, 446 (2010).

${ }^{48}$ J. Zhou, H. F. Pang, L. Garcia-Gancedo, E. Iborra, M. Clement, M. De Miguel-Ramos, H. Jin, J. K. Luo, S. Smith, S. R. Dong, D. M. Wang, and Y. Q. Fu, Microfluid. Nanofluid. 18, 537 (2015).

${ }^{49}$ S. Ippolito, K. Kalantar-Zadeh, D. Powell, and W. Wlodarski, in IEEE Symposium on Ultrasonics (IEEE, 2003), pp. 303-306.

${ }^{50}$ D. Royer and E. Dieulesaint, in Elastic Waves in Solids II: Generation, Acousto-optic Interaction, Applications, Advanced Texts in Physics (Springer, Berlin, Heidelberg, 2010).

${ }^{51}$ E. Lean and C. Powell, Proc. IEEE 58, 1939 (1970).

${ }^{52}$ R. Tucoulou, F. de Bergevin, O. Mathon, and D. Roshchupkin, Phys. Rev. B 64, 134108 (2001).

${ }^{53}$ R. Tucoulou, R. Pascal, M. Brunel, O. Mathon, D. V. Roshchupkin, I. a. Schelokov, E. Cattan, and D. Remiens, J. Appl. Crystallogr. 33, 1019 (2000).

${ }^{54}$ W. Sauer, M. Streibl, T. H. Metzger, a. G. C. Haubrich, S. Manus, A. Wixforth, J. Peisl, A. Mazuelas, J. Hartwig, and J. Baruchel, Appl. Phys. Lett. 75, 1709 (1999).

${ }^{55}$ L. Largeau, I. Camara, J.-Y. Duquesne, C. Gourdon, P. Rovillain, L. Thevenard, and B. Croset, J. Appl. Crystallogr. 49, 2073 (2016).

${ }^{56}$ J.-D. Nicolas, T. Reusch, M. Osterhoff, M. Sprung, F. J. R. Schülein, H. J. Krenner, A. Wixforth, and T. Salditt, J. Appl. Crystallogr. 47, 1596 (2014).

${ }^{57}$ M. Pernpeintner, "Magnon-phonon coupling in ferromagnetic thin films," Ph.D. thesis (Technische Universität München, 2012).

${ }^{58}$ M. Weiler, H. Huebl, F. S. Goerg, F. D. Czeschka, R. Gross, and S. T. B. Goennenwein, Phys. Rev. Lett. 108, 176601 (2012).

${ }^{59}$ F. E. Terman, Radio Engineer's Handbook (McGraw-Hill Book Company, Inc., 1943).

${ }^{60}$ R. I. Cottam and G. A. Saunders, J. Phys. C: Solid State Phys. 6, 2105 (1973).

${ }^{61}$ L. Thevenard, C. Gourdon, J. Y. Prieur, H. J. von Bardeleben, S. Vincent, L. Becerra, L. Largeau, and J.-Y. Duquesne, Phys. Rev. B 90, 094401 (2014). 
${ }^{62} \mathrm{P}$. Wright, in Proceedings of the 43rd Annual Symposium on Frequency Control (1989), p. 596.

${ }^{63} \mathrm{P}$. V. Wright, in Proceedings of the IEEE Ultrasonics Symposium (IEEE, 1989), p. 141.

${ }^{64} \mathrm{~T}$. Thorvaldsson, in Proceedings of the IEEE Ultrasonics Symposium (IEEE, 1989), pp. 91-96.

${ }^{65}$ A. Haddou, T. Gryba, J. E. Lefebvre, V. Sadaune, V. Zhang, and E. Cattan, in Proceedings of the IEEE Ultrasonics Symposium (2000), Vol. 1, pp. 91-94.

${ }^{66}$ V. Plessky and J. Kostela, Int. J. High Speed Electron. Syst. 10, 867 (2000).

${ }^{67}$ T.-T. Wu, S.-M. Wang, Y.-Y. Chen, T.-Y. Wu, P.-Z. Chang, L.-S. Huang, C.-L. Wang, C.-W. Wu, and C.-K. Lee, Jpn. J. Appl. Phys., Part 1 41, 6610 (2002).
${ }^{68}$ For a metallization ratio of 0.5 , the resistance of one finger electrode is $4 \rho_{\mathrm{Au}} l_{f} / h_{e} \lambda$ where $l_{f}$ is the length of the finger and $h_{e}$ is the metal thickness. For $N$ fingers connected to the same busbar ( $N$ shunt resistors) this gives an equivalent resistance of $4 \rho_{\mathrm{Au}} l_{f} / N h_{e} \lambda$ and, therefore, an equivalent total resistance of $8 \rho_{\mathrm{Au}} l_{f} / N h_{e} \lambda \simeq 8 \rho_{\mathrm{Au}} W / N h_{e} \lambda$ for our transducer.

${ }^{69}$ R. A. Matula, J. Phys. Chem. Ref. Data 8, 1147 (1979).

${ }^{70}$ K. S. Champlin, Appl. Phys. Lett. 12, 231 (1968).

${ }^{71}$ M. Clement, L. Vergara, J. Sangrador, E. Iborra, and A. Sanz-Hervás, Ultrasonics 42, 403 (2004).

${ }^{72}$ D. Frickey, IEEE Trans. Microwave Theory Tech. 42, 205 (1994).

${ }^{73}$ F. Kubat, W. Ruile, T. Hesjedal, J. Stotz, U. Rösler, and L. M. Reindl, IEEE Trans. Ultrason., Ferroelectr. Freq. Control 51, 1437 (2004).

${ }^{74}$ S. Ramo, J. R. Whinnery, and T. Van Duzer, in Fields and Waves in Communication Electronics, 3rd ed. (Wiley, New York, 1994). 